\title{
Digital Image Watermarking Algorithm Based on Fast Curvelet Transform
}

\author{
Jindong Xu, Huimin Pang, Jianping Zhao
}

College of Physical Engineering, Qufu Normal University, Shandong, China.

Email: xujindong1980@yahoo.com.cn

Received July $15^{\text {th }}, 2010$; revised August $18^{\text {th }}, 2010$; accepted August $21^{\text {st }}, 2010$.

\begin{abstract}
A digital image watermarking algorithm based on fast curvelet transform is proposed. Firstly, the carrier image is decomposed by fast curvelet transform, and, the watermarking image is scrambled by Arnold transform. Secondly, the binary watermarking image is embedded into the medium frequency coefficients according to the human visual characteristics and curvelet coefficients. Experiment results show that the proposed algorithm has good performance in both invisibility and security and also has good robustness against the noise, cropping, filtering, JPEG compression and other attacks.
\end{abstract}

Keywords: Digital Image Watermarking, Fast Curvelet Transform, Human Visual Characters, Robustness, Invisibility

\section{Introduction}

In the past two decades, more and more researchers have devoted to the transform domain which shows good performance and robustness. Recently, Candès and Donoho $[1,2]$ have developed a new multiscale transform which is called the Curvelet transform. It is anisotropic with strong direction, and provides optimally sparse representations of objects along a general curve with bounded curvature. Hence, it has been widely used in image processing field. At the same time, many research papers proposed to embed watermark based on the first curvelet transform [3-8].

Shi et al. [3] proposed a semi-fragile watermarking algorithm by embedding the wartermark in the maximum module of curvelet coefficient. This algorithm keeps good tolerance against JPEG compression. The method of Thai et al. [4] embedded a watermark in curvelet coefficients which are selected by a threshold. This method has good invisibility and robustness. Thai et al. [5] embedded the watermark in the curvelet transform which contain as much edge information as possible. It has good invisibility but poor robustness. The implementation of the first curvelet transform includes subband decomposition, smooth partitioning, renormalize and ridgelet analysis. So these algorithms have many problems such as implementation complexity and large amount of redundancy.
This paper proposes a digital image watermarking algorithm based on fast curvelet transform. Firstly, we take a binary-valued image which contains copyright information as watermark, and adapt Arnold transform to it to improve its security. Secondly, according to the human visual characteristics, we embed the watermark in curvelet coefficients of the original image. This algorithm is simple and easy to implement. Experiment results show that our algorithm has good invisibility and security, and is robustness to the noise, cropping, filtering, JPEG compression and other attacks.

\section{Curvelet Transform}

Similar to the wavelet transform and the ridgelet transform, the curvelet transform theory is based on sparsity theory [8]. The idea of curvelet is to calculate the inner relationship between the signal and the curvelet function to realize the sparse representation of the signal.

\subsection{Continuous-Time Curvelet Transform}

The curvelet transform can be expressed as

$$
c(j, l, k):=\left\langle f, \varphi_{j, l, k}\right\rangle
$$

here, $j=0,1,2, \ldots$, is a scale parameter; $l=0,1,2, \ldots$, is an orientation parameter; and $k=\left(k_{1}, k_{2}\right) \in \mathrm{Z}^{2}$ is a translation parameter. The mother curvelet is $\varphi_{j}(x)$, its Fourier transform is $\varphi_{j}(\omega)=U_{j}(\omega)$, where $U_{j}$ is fre- 
quency window defined in the polar coordinate system such as:

$$
U_{j}(r, \theta)=2^{-3 j / 4} W\left(2^{-j} r\right) V\left(\frac{2\lfloor j / 2\rfloor \theta}{2 \pi}\right)
$$

$W$ and $V$ are radial and angular windows respectively and will always obey certain admissibility conditions. Curvelet at scale $2^{-j}$, orientation $\theta_{l}$ and position $x_{k}^{(j, l)}=R_{\theta_{l}}^{-1}\left(k_{1} \times 2^{-j}, k_{2} \times 2^{-j}\right)$ can be expressed as:

$$
\phi_{j, l, k}(x)=\phi_{j}\left\lfloor R_{\theta_{l}}\left(x-x_{k}^{j, l}\right)\right\rfloor
$$

So, for $f \in L^{2}\left(R^{2}\right)$, curvelet transform is expressed as:

$$
\begin{aligned}
c(i, l, k) & :=\frac{1}{2 \pi^{2}} \int \hat{f}(\omega) \overline{\hat{\phi}}_{j, l, k}(\omega) \mathrm{d} \omega \\
& =\frac{1}{2 \pi^{2}} \int \hat{f}(\omega) U_{j}\left(R_{\theta_{l}} \omega\right) \exp \left(\mathrm{i}\left\langle x_{k}^{j, l}, \omega\right\rangle\right) \mathrm{d} \omega
\end{aligned}
$$

\subsection{Digital Curvelet Transform}

Digital curvelet transform is linear and takes as input Cartesian arrays of the form $f\left[t_{1}, t_{2}\right], 0 \leq t_{1}, t_{2}<n$, which allows the output as a collection of coefficients:

$$
c^{D}(j, l, k):=\sum_{0 \leq t_{1}, t_{2}<n} f\left[t_{1}, t_{2}\right] \overline{\varphi_{j, l, k}^{D}\left[t_{1}, t_{2}\right]}
$$

In order to improve the curvelet transform-in the sense that they are conceptually simpler, faster and far less redundant. Paper [2] proposed the Fast Discrete Curvelet Transform (FDCT). There are two digital implementation of FDCT. The first is based on unequally-spaced fast Fourier transform (USFFT) while the second is based on the wrapping of specially selected Fourier samples. The FDCT-Wrapping uses simpler choice of spatial grid to translate curvelets at each scale and angle. It needs less two-dimensional FFTs than FDCT-USFFT, so it is quickly.

The architecture of FDCT via Wrapping is then roughly as follows:

1) Apply the 2D FFT and obtain Fourier sample $\hat{f}\left[n_{1}, n_{2}\right],-n / 2 \leq n_{1}, n_{2} \leq n / 2$ ( $n$ is the size of the picture).

2) For each scale/angle pair $(j, l)$, form the product $\tilde{U}_{j, l}\left[n_{1}, n_{2}\right] \hat{f}\left[n_{1}, n_{2}\right]$.

3) Wrap this product around the origin and obtain $\tilde{f}_{j, l}\left[n_{1}, n_{2}\right]=W\left(\tilde{U}_{j, l} \hat{f}\right)\left[n_{1}, n_{2}\right]$, where the range for $n_{1}$ and $n_{2}$ is now $0 \leq n_{1}<L_{1, j}$ and $0 \leq n_{2}<L_{2, j}$ (for $\theta$ in the range $(-\pi / 4, \pi / 4)$.

4) Apply the inverse 2D FFT to each $\tilde{f}_{j, l}$, hence collecting the discrete coefficients $c^{D}(j, l, k)$.

\section{Watermarking Algorithm Based on FDCT}

\subsection{Watermark}

In this algorithm, the watermark is a binary-valued image with $32 \times 32$ pixels (Figure 1).

Arnold transform is used to increase the data security, and its function is defined as (6).

$$
\left(\begin{array}{l}
x^{\prime} \\
y^{\prime}
\end{array}\right)=\left(\begin{array}{cc}
1 & 1 \\
k & k+1
\end{array}\right)\left(\begin{array}{l}
x \\
y
\end{array}\right) \bmod N
$$

Adapt $n$ (here, $n=8$ ) times Arnold transform to the original watermark $\mathrm{W}$, then we obtain scrambling watermark (Figure 2).

\subsection{Watermark Embedding}

According to the Human Visual Characteristic, we choose to embed the watermark into medium-frequency. In selected scale, the coefficients of each orientation are sorted and find criterion $T_{i}$ by amplitude factor $\lambda(\lambda \geq 0)$. Then choose the minimum $T_{i}$ as embedding parameter $T$. Finally, the watermark is embedded to coefficients which are chosen by $T$.

The embedding procedure (Figure $\mathbf{3}$ ) is described as follows.

1) Apply FDCT to the original image, get curvelet coefficient $C$.

2) Select the curvelet coefficient to embed a bit watermark according to the following conditions.

For $0 \quad 0<C(j, l, k)<T / 2$ or $T<C(j, l, k)<3 T / 2$

For $1 T / 2<C(j, l, k)<T$

Then record the positions of these coefficients and the positions of 0 and 1 in the watermark.

3) The embedded coefficients are modified by the following equations.

$$
\begin{aligned}
\text { Embed } 0 \quad C^{\prime}(j, l, k)= & C(j, l, k) \\
& -\bmod (C(j, l, k), T)+T / 4 \\
\text { Embed } 1 \quad C^{\prime}(j, l, k)= & C(j, l, k) \\
& -\bmod (C(j, l, k), T)+3 T / 4
\end{aligned}
$$

4) Do inverse FDCT to $C$, obtain the watermarked image.

\subsection{Watermark Extraction}

The extraction procedure is composed of 4 steps and each step is described as follows.

\section{a b \\ c d}

Figure 1. Watermark.

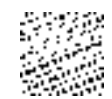

Figure 2. Scrambled watermark. 


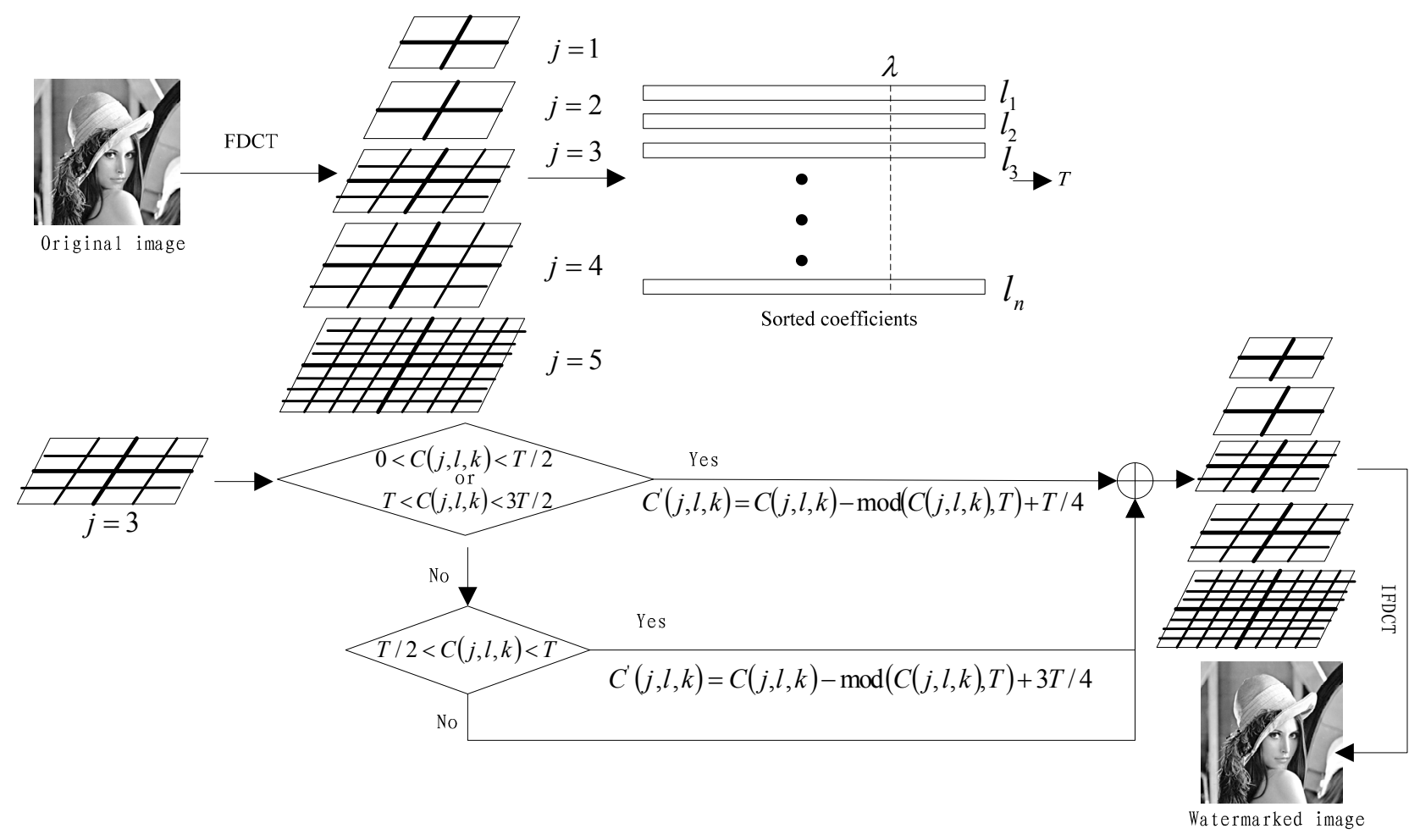

Figure 3. Embedding procedure.

1) Apply curvelet transform to watermarked image, obtain curvelet coefficient $C^{\prime}$.

2) Locate the watermark positions from the original image by using embedding procedure step 2 . Then extract the coefficients $C^{\prime}$ from the watermarked image using those watermark position.

3) Extract the watermark W' from C' with the following rule

$$
\omega_{i}=\left\{\begin{array}{lll}
1 & \text { if } & \bmod \left(C^{\prime}(l, j, k), T\right) \geq T / 2 \\
0 & \text { if } & \bmod \left(C^{\prime}(l, j, k), T\right)<T / 2
\end{array}\right.
$$

4) Apply $T^{\prime}-n$ ( $T^{\prime}$ is Arnold transform period) times Arnold transform to $\mathrm{W}^{\prime}$, obtain the binary watermark image.

\section{Experimental Results}

In this program, the image is transformed through FDCT via Wrapping. We use standard $512 \times 512$ pixel image 'Lena' (Figure 4) for evaluation of our proposed method, and conduct experiments binary-valued image watermarking with the noted above parameter scale $=3, \lambda=$ 0.75 .

We have investigated the invisibility and the robustness of our watermarking system, analyzed the algorithm performance by objective and subjective standards.

\subsection{Invisibility Tests}

The watermarked image is shown in Figure 5(a), the detected watermark from watermarked image is shown in Figure 5(b).

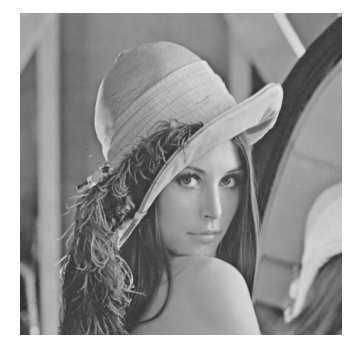

Figure 4. Original image.

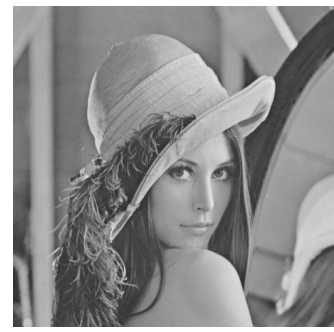

(a)

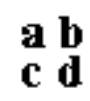

(b)
Figure 5. Invisibility Tests. (a) Watermarked image [PSNR $=60.8028 \mathrm{~dB}]$; (b) Detected watermark $[\mathrm{NC}=1.00]$. 
Table 1. JPEG compression attack.

\begin{tabular}{|c|c|c|c|c|c|c|c|c|c|}
\hline$Q$ & 90 & 80 & 70 & 60 & 50 & 40 & 30 & 20 & 15 \\
\hline PSNR & 25.2093 & 25.1357 & 25.0710 & 25.0186 & 24.9718 & 24.8890 & 24.8226 & 24.6714 & 24.4813 \\
\hline $\mathrm{NC}$ & 0.9988 & 0.9975 & 0.9901 & 0.9877 & 0.9914 & 0.9741 & 0.9532 & 0.9470 & 0.9113 \\
\hline $\begin{array}{l}\text { Detected } \\
\text { watermark }\end{array}$ & $\begin{array}{l}a b \\
c d\end{array}$ & $\begin{array}{l}\dot{a} \mathbf{b} \\
\mathbf{c} d\end{array}$ & $\begin{array}{c}a b \\
c\end{array}$ & $\begin{array}{l}\mathbf{a} \\
\mathbf{c}\end{array}$ & $\begin{array}{l}\mathrm{a} \\
\mathrm{c}\end{array}$ & $\begin{array}{l}b \\
\mathbf{c}\end{array}$ & $\mathrm{ad}$ & $\mathrm{di}$ & मt: \\
\hline
\end{tabular}

Table 2. Gaussian low pass filtering attack.

\begin{tabular}{|c|c|c|c|c|c|}
\hline $\begin{array}{l}\text { Standard Deviation } \\
\text { (Window })\end{array}$ & $0.5(3)$ & $1.5(3)$ & $0.5(5)$ & $1.5(5)$ & $3(5)$ \\
\hline PSNR & 40.8289 & 32.4277 & 40.8027 & 29.9117 & 28.7562 \\
\hline $\mathrm{NC}$ & 0.9914 & 0.9704 & 0.9914 & 0.9113 & 0.8658 \\
\hline $\begin{array}{l}\text { Detected } \\
\text { watermark }\end{array}$ & $\begin{array}{l}a b \\
c b\end{array}$ & $\begin{array}{l}\mathbf{b} \\
\mathrm{d} \cdot \mathrm{d}\end{array}$ & $\begin{array}{l}a \\
c \\
c\end{array}$ & $a b$ & $9 \mathrm{~d}$ \\
\hline
\end{tabular}

Table 3. Other attacks.

\begin{tabular}{|c|c|c|c|c|c|}
\hline Attacks & $\begin{array}{c}\text { Gaussian noise } \\
(0.001)\end{array}$ & $\begin{array}{c}\text { Salt \& Pepper noise } \\
(0.01)\end{array}$ & $\begin{array}{c}\text { Random cropping } \\
(1 / 16)\end{array}$ & $\begin{array}{c}\text { Random cropping } \\
(1 / 32)\end{array}$ & Change contrast \\
\hline PSNR & 19.4926 & 25.0252 & 19.6377 & 21.7762 & 25.2690 \\
\hline $\mathrm{NC}$ & 0.8116 & 0.9200 & 0.8645 & 0.9372 & 0.8756 \\
\hline $\begin{array}{l}\text { Detected } \\
\text { watermark }\end{array}$ & काष & ab: & $\mathrm{CH}$ & $\mathrm{d}$ & $\alpha d$ \\
\hline
\end{tabular}

Subjectively, the watermarked image has good invisibility. Compare Figure 5(b) with Figure 2, they are fully consistent. The original watermark is accurately recovered.

\subsection{Robustness Tests}

To evaluate the robustness of algorithm, all the attacks are tested by the software of Stirmark [10,11]. The Peak Signal to Noise Ratio (PSNR) is employed to evaluate the quality of watermarked image after attack, and the Normalized Correlation Coefficient (NC) is used to evaluate the quality of extracted watermark for some attacks such as JPEG compression (Quality factor Q), Gaussian low pass filtering, adding noise, cropping and so on. The simulation results are shown as following Tables.

It can be seen from Table 1 and Table 2, the proposed method show very good robustness to JPEG compression and Gaussian low pass filtering. From Table 3, the method also has good robustness against noise, cropping and so on.

\section{Conclusions}

This paper proposes a method by embedding a watermark into the original image based on FDCT. At the same time, Arnold transform is applied to improve the security of the system. The experimental results show that the watermarked image has good invisibility and robustness against JPEG compression and Gaussian low pass filtering.

There are many unstable coefficients are discovered from the experiments. For instance, a small change of the image will arouse big changes of these coefficients. These unstable factors can influence the extracting of watermark. Therefore, the proposed algorithm can not give a good performance of rotation and scaling, etc. In the future, we will unceasing devote ourselves to the study of the robustness watermark system against geometric attacks based on curvelet transform.

\section{REFERENCES}

[1] D. L. Donoho and M. R. Duncan, "Digital Curvelet 
Transform: Strategy, Implementation and Experiments," Proceeding of the SPIE on Wavelet Application VII, Orlando, 2000, pp. 12-30.

[2] E. J. Candes, L. Demanet and D. L. Donoho, "Fast Discrete Curvelet Transforms [R]," Applied and Computational Mathematics, 2005, pp. 1-43.

[3] J. P. Shi and Z. J. Zhai, "Curvelet Transform for Image Authentication," Rough Set and Knowledge Technology, LNAI 4062, 2006, pp. 659-664.

[4] T. D. Hien, I. Kei, H. Harak, et al., "Curvelet-Domain Image Watermarking Based on Edge-Embedding," Lecture Notes in Computer Science, Vol. 4693, No. 2010, 2007, pp. 311-317.

[5] T. D. Hien, K. Miyara, et al., "Curvelet Transform Based Logo Watermarking," Innovative Algo-Rithms and Techniques in Automation, Industrial Electronics and Telecommunication, 2007, pp. 305-309.

[6] H. Y. Leung, L. M. Cheng and L. L. Cheng, "Digital Watermarking Schemes Using Multi-Resolution Curvelet and HVS Model," Lecture Notes in Computer Science, Vol. 5703, 2009, pp. 4-13.

[7] H. Y. Leung, L. M. Cheng and L. L. Cheng. "A Robust Watermarking Scheme Using Selective Curvelet Coefficients," International Conference on Intelligient Information Hiding and Multimedia Signal Processing, Harbin, August 2008, pp. 465-468.

[8] T. D. Hien, K. Miyara, I. Kei and F. F. Ali, "Digital Watermarking Based on Curvelet Transform," 9th International Symposium on Signal Processing and Its Applications, Sharjah, Feburary 2007, pp. 1-4.

[9] J. L. Cheng and T. Shan. "Development and Prospect of Image Multiscale Geometric Analysis," Acta Electronic Sinica, Vol. 31, No. 12 A, pp. 1975-1981.

[10] A. P. Fabien and Petitcolas. "Watermarking Schemes Evaluation," IEEE. Signal Processing, Vol. 17, No. 5, 2000, pp. 58-64.

[11] Stirmark. http://www.petitcolas.net/fabien/watermarking /stirmark/ 